\title{
Increased left atrial chamber stiffness in hypertrophic cardiomyopathy
}

Hiroto Sanada, Masami Shimizu, Norihiko Sugihara, Kuniyoshi Shimizu, Hidekazu Ino, Ryoyu Takeda

\begin{abstract}
Objective-To investigate left atrial chamber stiffness and its influence on left atrial and left ventricular functions in hypertrophic cardiomyopathy.

Design-Prospective study.
\end{abstract}

Setting-Department of internal medicine in a university teaching hospital.

Patients-Five control subjects, six patients with essential hypertension, and 11 patients with hypertrophic cardiomyopathy.

Interventions-Measurement of left atrial pressure by a tip micromanometer and of real-time left atrial volume from left atrial cineangiograms.

Main outcome measure-Left atrial stiffness constant determined by fitting the ascending limb of the $v$ loop of the left atrial pressure-volume relation to an exponential curve.

Results-The mean (SD) left atrial chamber stiffness constant was significantly larger in patients with hypertrophic cardiomyopathy than in controls $(0.063$ $(0.018) v 0.041(0.006), p<0.05)$ and was correlated with left ventricular wall thickness $(r=0.560, p<0.01)$. Left atrial reservoir volume (left atrial emptying volume before atrial contraction) was significantly smaller in patients with hypertrophic cardiomyopathy than in the controls $\left(7 \cdot 3(2 \cdot 1) v 12.5(4 \cdot 4) \mathrm{ml} / \mathrm{m}^{2}, \mathrm{p}<\right.$ $0.01)$ and was inversely correlated with the left atrial chamber stiffness constant $(r=-0.598, p<0.01)$. The cardiac index was inversely correlated with the left atrial chamber stiffness constant $(r=$ $-0.542, p<0.01$ ).

Conclusions-Left atrial chamber stiffness was increased in patients with hypertrophic cardiomyopathy and this affected the left atrial reservoir function. This may in turn have affected cardiac output.

\section{(Br Heart J 1993;69:31-35)}

The left atrium functions as a reservoir, a conduit, and a booster pump for left ventricular filling. ${ }^{\prime}$ Many investigations have emphasised the booster pump function, whereas the left atrium is also important as a reservoir, accepting venous return while the mitral valve is shut Grant et $a l^{2}$ showed that the energy stored during left atrial filling is much greater than left atrial work during atrial contraction. Using a circulatory analogue model Suga showed an improvement in cardiac performance with larger atrial compliances. ${ }^{3}$

Left atrial distensibility has been reported in various disease states. For example, in a canine model of mitral regurgitation, increased left atrial compliance prevented the rise in left atrial pressure. ${ }^{4}$ Increased left atrial stiffness was shown in patients with hypertension. ${ }^{5}$

The pathophysiology of hypertrophic cardiomyopathy is characterised by increased impedance to diastolic filling with increased ventricular stiffness. Although the left atrium is important in ventricular filling, the importance of left atrial stiffness in patients with hypertrophic cardiomyopathy is uncertain. We investigated left atrial chamber stiffness and its influence on cardiac performance in patients with hypertrophic cardiomyopathy by analysing the left atrial pressure-volume loops.

\section{Patients and methods}

PATIENTS

We studied a control group of five patients with chest pain in whom investigation, including cardiac catheterisation and angiography, showed no abnormality. We also studied six patients with World Health Organisation grades I and II essential hypertension and 11 patients with hypertrophic cardiomyopathy. There were no significant differences in age or sex distribution between the three groups (table 1). All patients were in normal sinus rhythm. We excluded patients with coexisting angiographically confirmed significant coronary artery stenosis or spasm, diabetes mellitus or other endocrine disease, and those with other disorders affecting cardiac function. All patients gave informed consent to the study. There were no complications attributable to the investigative procedures.

\section{CARDIAC CATHETERISATION AND ANGIOGRAPHY}

All medications were stopped for two weeks before catheterisation. We measured intracardiac pressures in the right heart, cardiac output, aortic pressure and left atrial and ventricular pressures. Then left atrial cineangiography, left ventriculography, biventriculography, and coronary angiography were performed.

Left-sided intracardiac pressures were measured with a 4 French transducer-tipped pressure monitoring catheter (Model 110-4, Camino Laboratories; San Diego, CA, USA) and a digital pressure monitor (Camino 420). To adjust for the zero shift caused by changes 
Table 1 Clinical characteristics and haemodynamic variables in the three groups (mean $(S D)$ )

\begin{tabular}{llll}
\hline Variable & Control & Hypertension & $\begin{array}{l}\text { Hypertrophic } \\
\text { cardiomyopathy }\end{array}$ \\
\hline Number of patients & 5 & 6 & 11 \\
Male/female & $4 / 1$ & $5 / 1$ & $8 / 3$ \\
Age (yr) & $57(17)$ & $57(7)$ & $58(17)$ \\
Left ventricular wall thickness (mm) & $19(2)$ & $28(4) \dagger$ & $34(6) \dagger$ \\
Interventricular septal thickness (mm) & $10(1)$ & $15(2) \dagger$ & $20(4) \dagger \ddagger$ \\
Left ventricular relaxation time constant (ms) & $43(7)$ & $49(5)$ & $58(11)^{\star}$ \\
Left ventricular ejection fraction (\%) & $73(7)$ & $70(7)$ & $64(14)$ \\
\hline
\end{tabular}

${ }^{\star} \mathrm{p}<0.05$ and $+\mathrm{p}<0.01$ compared with control group.

$\mathrm{p}<0.01$ compared with hypertension group.

in measurement position and temperature during the procedure, pressures were measured simultaneously with a fluid-filled catheter. Left atrial and ventricular pressures were recorded at a paper speed of $250 \mathrm{~mm} / \mathrm{s}$.

Left atrial pressure was measured by introducing a tip-micromanometer into the left atrium through a transseptal Brockenbrough catheter or through a pigtail 8 French catheter inserted from the left ventricle. Left atrial cineangiography was carried out by injecting $45 \mathrm{ml}$ of contrast medium (iopamidol, Schering) into the pulmonary artery at a rate of $15 \mathrm{ml} / \mathrm{s}$. Bi-plane cineangiograms (anteroposterior and lateral projections) were obtained at $60 \mathrm{frames} / \mathrm{s}$. Left atrial pressure was recorded during left atriography. Cineangiograms were performed during shallow expiration to avoid changes in intracardiac pressure and volume induced by respiration.

Left ventriculography was carried out by injecting $35 \mathrm{ml}$ of contrast medium into the left ventricle at a rate of $10 \mathrm{ml} / \mathrm{s}$ bi-directionally in the $30^{\circ}$ right anterior oblique and $60^{\circ}$ left

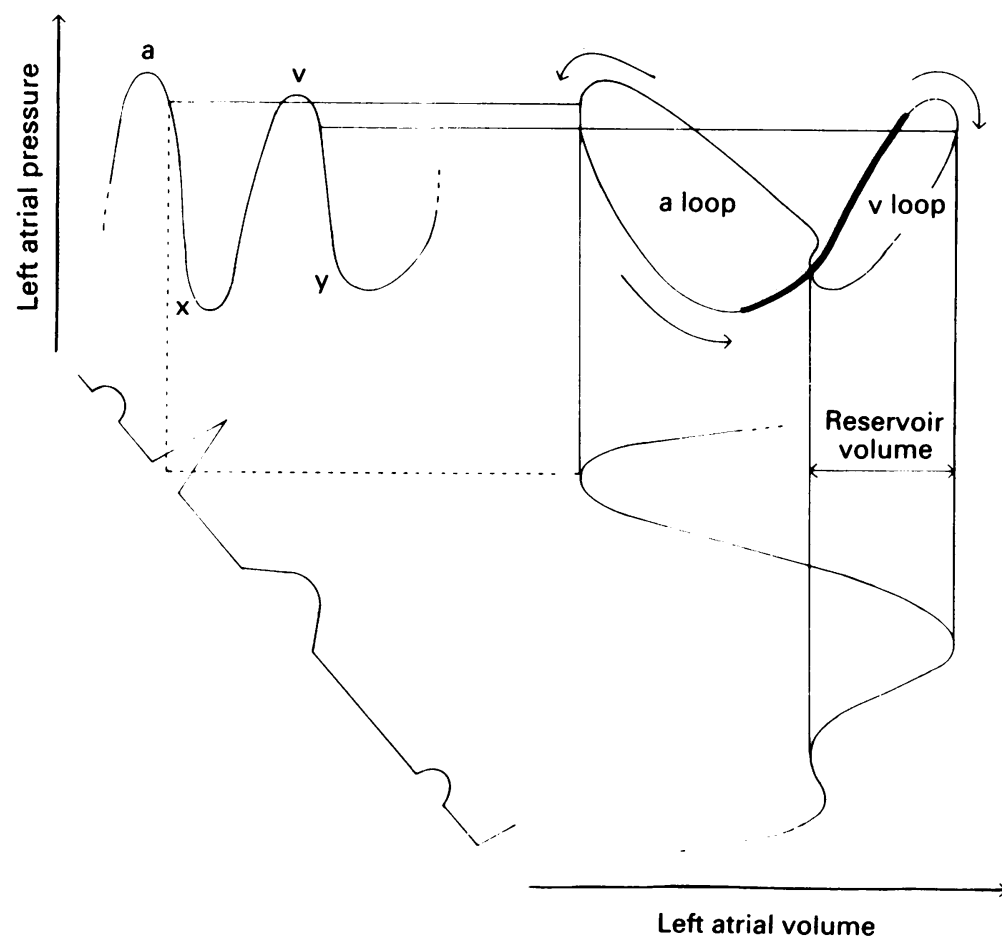

Figure 1 Left atrial pressure-volume loop obtained from left atrial volume-time curve (horizontal axis) and simultancously measured left atrial pressure curve (vertical axis). (horizontal axis) and simultaneously measured left atrial pressure curve (vertical axis)
The pressure-volume loop consists of an a loop on the left and a v loop on the right. $a, a$ wave of left atrial pressure; $v, v$ wave of left atrial pressure; $x, x$ descent of left atrial pressure; $y, y$ descent of left atrial pressure. anterior oblique projections. During left ventriculography left ventricular pressure was simultaneously recorded.

Bi-ventriculography was carried out by injecting $15 \mathrm{ml}$ of contrast medium into the right ventricle and $25 \mathrm{ml}$ into the left ventricle simultaneously at a rate of $10 \mathrm{ml} / \mathrm{s}$ in the left anterior projection. This showed the endocardial surfaces of the ventricular septum and posterior wall.

\section{DATA ANALYSIS}

Frame by frame left atrial volumes were calculated with an MSR Corporation model Sigma-5/E picture analyser, ${ }^{67}$ and the left atrial time-volume curve was plotted by Fourier analysis. The left atrial pressure-volume loop was obtained from the left atrial time-volume curve and the real time left atrial pressure curve $^{289}$ (fig 1). The left atrial pressure-volume relation in the ascending limb of the $\mathrm{v}$ loop was fitted to an exponential function $p=b e^{K V}$, where $P=$ left atrial pressure, $V=$ left atrial volume, $\mathrm{b}=$ intercept, $\mathrm{K}=$ coefficient, and $\mathrm{e}=$ the base of the natural logarithm. The coefficient $\mathrm{K}$ is the left atrial chamber stiffnes constant. ${ }^{510}$ As an index of left atrial reservoir function we determined the left atrial emptying volume before atrial contraction and used it as a measure of the left atrial reservoir volume. ${ }^{11} \mathrm{We}$ measured the flow volume from the pulmonary vein into the left ventricle (left atrial conduit volume) and used it as an index of left atrial conduit function. ${ }^{\text {" }}$ The left atrial conduit volume equals the left atrial stroke volume subtracted from the left ventricular stroke volume.

The left ventricular pressure-volume relation was obtained from frame by frame left ventricular volumes and real-time left ventricular pressure curves obtained during left ventriculography. The left ventricular pressure-volume relation during the late diastolic phase was fitted to an exponential function $P=b e^{K V}$, where $P=$ left ventricular pressure, $\mathrm{V}=$ left ventricular volume, $\mathrm{b}=$ intercept, $\mathrm{K}=$ left ventricular chamber stiffness constant, and $e=$ the base of the natural logarithm. ${ }^{1213}$ We used the left ventricular chamber stiffness constant as an index of left atrial afterload.

For left ventricular wall thickness we measured the sum of the interventricular septal thickness and left ventricular posterior wall thickness from the bi-ventriculogram. ${ }^{14}$ The time constant $T$ for the relaxation phase pressure fall ${ }^{15}$ was determined from the left ventricular pressure.

\section{STATISTICAL ANALYSIS}

Data from the three groups were compared by the one-way layout analysis of variance. For factors showing significant differences, data from two groups were compared by Student's unpaired $t$ test. Correlations between each factor were determined by Pearson's correlation. Statistical significance was defined as $\mathrm{p}<0.05$. 
Table 2 Left atrial chamber stiffness constant and reservoir volume index in the three groups (mean $(S D)$ )

\begin{tabular}{llll}
\hline Variable & Control & Hypertension & $\begin{array}{l}\text { Hypertrophic } \\
\text { cardiomyopathy }\end{array}$ \\
\hline Left atrial chamber stiffness constant & $0.041(0 \cdot 006)$ & $0.055(0 \cdot 016)$ & $0.063(0 \cdot 018)^{\star}$ \\
Left atrial reservoir volume index $\left(\mathrm{ml} / \mathrm{m}^{2}\right)$ & $12(4)$ & $9(3)$ & $7(2) \dagger$ \\
\hline
\end{tabular}

${ }^{\star} \mathrm{p}<0.05$ and $+\mathrm{p}<0.01$ compared with control group.

\section{Results}

LEFT VENTRICULAR WALL THICKNESS AND INDICATORS OF CIRCULATORY FUNCTION

(TABLE 1)

Left ventricular wall thickness was significantly greater in the groups with hypertension and hypertrophic cardiomyopathy than in the control group. The interventricular septal thickness was significantly greater in the hypertrophic cardiomyopathy group than in the other two groups. The left ventricular relaxation time constant was significantly longer in the hypertrophic cardiomyopathy group than in the control group. The left ventricular ejection fraction was similar in the three groups.

\section{LEFT ATRIAL CHAMBER STIFFNESS \\ (TABLE 2, FIGURE 2)}

Compared with the control group the left atrial chamber stiffness constant tended to be greater in the hypertension group and was significantly greater in the hypertrophic cardiomyopathy group. The left ventricular wall thickness correlated with the left atrial chamber stiffness constant.

\section{LEFT ATRIAL RESERVOIR FUNCTION \\ (TABLE 2, FIGURE 3)}

Compared with the control group the left atrial reservoir volume tended to be decreased in the hypertension group and was significantly decreased in the hypertrophic cardiomyopathy group. There was a negative correlation between the left atrial chamber stiffness constant and the left atrial reservoir volume.

LEFT ATRIAL CONDUIT FUNCTION (FIGURE 4) There was a negative correlation between the left ventricular chamber stiffness constant and the left atrial conduit volume in the combined

Figure 2 Relation between left ventricular wall thickness and left atrial chamber stiffnes constant. The regression line for the three groups is shown.

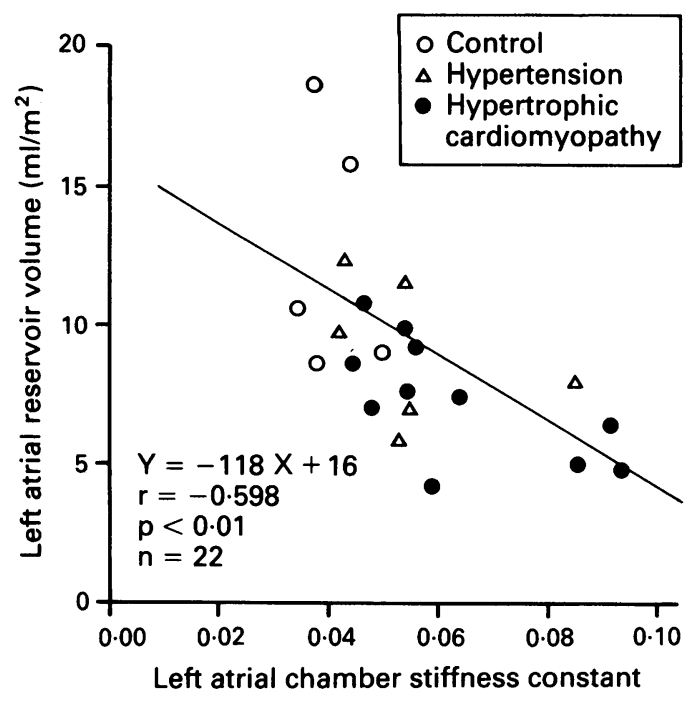

Figure 3 Relation between left atrial chamber stiffness constant and left atrial reservoir volume. The regression line for the three groups is shown.

control and hypertension groups. In the hypertrophic cardiomyopathy group, those patients with greatly increased left ventricular chamber stiffness had values to the upper right of the regression line.

RELATION BETWEEN LEFT ATRIAL CHAMBER STIFFNESS AND CARDIAC INDEX (FIGURE 5)

There was a negative correlation between the left atrial chamber stiffness constant and the cardiac index.

\section{Discussion}

The left atrium is positioned at the inlet to the left ventricle and functions as a reservoir for blood returning from the pulmonary vein during left ventricular systole. Additionally, the left atrium functions as a conduit for left ventricular filling. During atrial systole the left atrium functions as a pump which actively

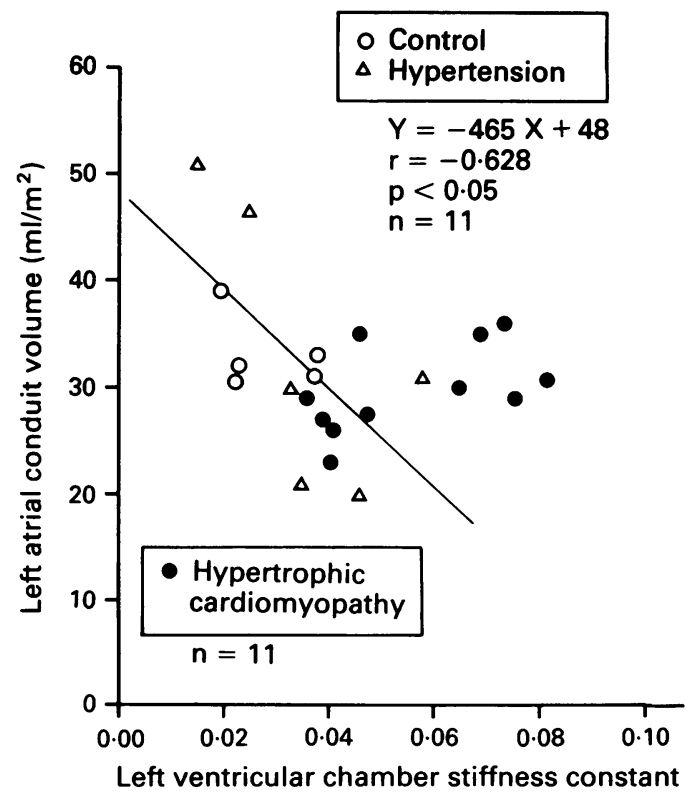

Figure 4 Relation between left ventricular chamber stiffness constant and left atrial conduit volume. The regression line for the combined control and hypertension groups is shown. 
Figure 5 Relation between left atrial chamber stiffness constant and cardiac index. The regression line for the three groups is shown.

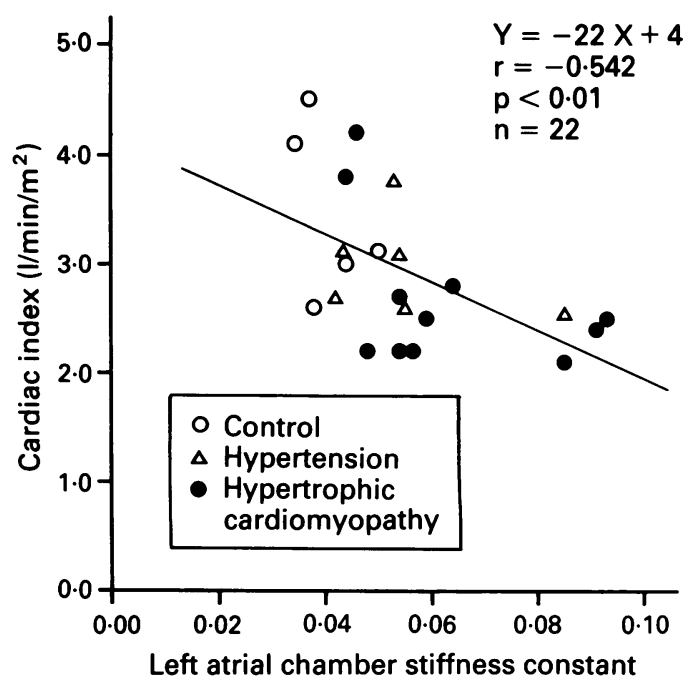

ejects blood into the left ventricle. The left atrial pressure-volume loop is made up of an a loop and a $\mathrm{v}$ loop. The a loop represents left atrial systolic function and the $\mathrm{v}$ loop left atrial reservoir function. Left atrial chamber stiffness is obtained by fitting the left atrial pressurevolume relation in the ascending limb of the $v$ loop to an exponential function.

The left atrial chamber stiffness constant tended to be greater in the hypertension group and was significantly greater in the hypertrophic cardiomyopathy group than in the control group. Both left atrial chamber stiffness and muscle stiffness were higher in spontaneously hypertensive rats ${ }^{16}$ and patients with hypertension. ${ }^{5}$ Left atrial stiffness in hypertrophic cardiomyopathy, however, has yet to be investigated. Increased left atrial chamber stiffness can be caused by an increase in left atrial muscle stiffness and/or an increase in left atrial wall thickness. ${ }^{17}$ It is difficult, however, to determine the left atrial wall thickness with presently available clinical methods. Further investigation is required.

There was a positive correlation between the left ventricular wall thickness and the left atrial chamber stiffness constant. Some of the increase in left atrial stiffness may be attributable to changes in left atrial myocardial function caused by an increase in left atrial afterload, ${ }^{18}$ which in turn is caused by left ventricular hypertrophy. ${ }^{19}$ Additionally, it has been suggested that intrinsic muscle disease in hypertrophic cardiomyopathy affects the atrial muscle itself. ${ }^{20}$

There was a negative correlation between the left atrial chamber stiffness and the left atrial reservoir volume. In patients with hypertrophic cardiomyopathy the left atrial reservoir function is reduced by the increase in left atrial chamber stiffness. We suggest that the left atrial stiffness affects left ventricular filling.

Investigation of the left atrial conduit function showed that the left atrial conduit volume was decreased by the increased left ventricular chamber stiffness in patients with hypertension. Because the conduit volume is a passive flow volume from the pulmonary vein into the left ventricle, it depends on left ventricular stiff- ness. ${ }^{11}$ In the hypertrophic cardiomyopathy group, however, values lay to the upper right of the regression line for the other two groups. In patients with hypertrophic cardiomyopathy, the conduit function seems to be augmented to compensate for the reduced reservoir function. $\underline{\text { W }}$ The increase in left atrial stiffness in patients $I$ with hypertrophic cardiomyopathy may augment left ventricular filling during the slow ${ }_{C}$ filling period in the face of increased left $\overrightarrow{\vec{F}}$ ventricular stiffness. ${ }^{10}$

We found a negative correlation between left 0 atrial stiffness and cardiac index. The data $\frac{\bar{F}}{\bar{\omega}}$. suggest that increased left atrial stiffness affects $\frac{\bar{\sigma}}{\vec{\sigma}}$ left ventricular performance. This is consistent $\varnothing$ with the suggestion that the stiff left atrium $\%$ reduces the cardiac output by reducing $\overrightarrow{0}$ pulmonary venous return. ${ }^{3}$ Atrial fibrillation is believed not to affect cardiac output to any great $\omega_{\sigma}$ extent because the reservoir function is maintained even when the atrial pump function is lost. ${ }^{221}$ Atrial fibrillation can cause profound haemodynamic dysfunction in some patients $\vec{\omega}$ with hypertrophic cardiomyopathy if reservoir 0 function is reduced. ${ }^{22}$

We measured left atrial chamber stiffness in $\vec{c}$ controls and patients with hypertension and hypertrophic cardiomyopathy and investigated the influence of this index on left atrial and left ventricular functions. Left atrial chamber stiff- $\overrightarrow{0}$ ness tended to be reduced in patients with $\omega$ hypertension and was significantly reduced in 0 patients with hypertrophic cardiomyopathy. This increase in left atrial stiffness was influenced by increased left ventricular wall thickness and resulted in a decrease of the left $\stackrel{\mathbb{Q}}{\mathscr{Q}}$ atrial reservoir volume. In patients with hyper- $\overrightarrow{\overrightarrow{\vec{O}}}$ trophic cardiomyopathy the conduit function 3 of the left atrium seemed to be augmented. Increased left atrial stiffness was associated with a decrease in the cardiac index. We conclude that left atrial chamber stiffness is increased in hypertrophic cardiomyopathy and that it may affect cardiac output by reducing left atrial reservoir function.

1 Leonard JJ, Shaver J, Thompson M. Left atrial transport function. Trans Am Clin Climatol Assoc 1980;92:133-41. 2 Grant C, Bunnell IL, Greene DG. The reservoir function of the left atrium during ventricular systole. An angiocar- $N$ diographic study of atrial stroke volume and work. Am J N
Med 1963;37:36-43.

3 Suga $\mathrm{H}$. Importance of atrial compliance in cardiac perfor- $\sigma$ mance. Circ Res 1974;35:39-43.

4 Kihara Y, Sasayama S, Miyazaki S, et al. Role of the left atrium in adaptation of the heart to chronic mitral $\frac{}{\Phi}$ regurgitation in conscious dogs. Circ Res 1988;62:543-53. \&

5 Natsume T, Nakajima K, Shibata $H$, et al. Left ventricular : and left atrial function in patients with borderline and and left atrial function in patients with borderline and

6 Sauter HJ, Doge H'T, Johnston RR, Graham 'T. The relationship of left atrial pressure and volume in patients relationship of left atrial pressure and volume in
with heart disease. Am Heart $J$ 1964;67:635-42.

7 Murray JA, Kennedy JW, Figley MM. Quantitative angiocardiography. II. 'The normal left atrial volume in man. Circulation 1968;37:800-4.

8 Sasayama S, Takahashi M, Osakada G, et al. Dynamic geometry of the left atrium and left ventricle in acute mitral regurgitation. Circulation 1979;60:177-86.

9 Matsuda Y, Toma Y, Ogawa $\mathrm{H}$, et al. Importance of left atrial function in patients with myocardial infarction. Circulation 1983;67:566-71.

10 Nagano 'T, Arakawa M, Tanaka 'T', et al. Diastolic compliance of the left atrium in man: a determinant of preload of the left ventricle. Heart Vessels 1989;5:25-32.

11 Matsuda Y, Toma Y, Moritani K, ct al. Assessment of left atrial function in patients with hypertensive heart discase. Hypertension 1986;8:779-85. 
12 Gaasch WH, Battle WE, Oboler AA, Banas JS Jr, Levine HJ. Left ventricular stress and compliance in man. With special reference to normalized ventricular function curves. Circulation 1972;45:746-62.

13 Barry WH, Brooker JZ, Alderman EL, Harrison DC Changes in diastolic stiffness and tone of left ventricle during angina pectoris. Circulation 1974;49:255-63.

14 Redwood DR, Scherer JL, Epstein SE. Biventricular cineangiography in the evaluation of patients with cineangiography in the evaluation of patients with
asymmetric septal hypertrophy. Circulation 1974;49: asymmetric

15 Weiss JL, Frederiksen JW, Weisfeldt ML. Hemodynamic determinants of the time-course of fall in canine left ventricular pressure. J Clin Invest 1976;58:751-60.

16 Ricksten S-E, Yao T, Ljung B, Thoren P. Distensibility of left atrium in normotensive and spontaneously hypertensive rats. Acta Physiol Scand 1980;110:413-18.

17 Gaasch WH, Levine HJ, Quinones MA, Alexander JK. Left ventricular compliance: mechanisms and clinical implications. Am J Cardiol 1976;38:645-53.

18 Mercadier J-J, Bastie D, Ménasché P, et al. Alpha-myosin heavy chain isoform and atrial size in patients with various types of mitral valve dysfunction: a quantitative study. $J$ Am Coll Cardiol 1987;9:1024-30.

19 Grossman W, McLaurin LP, Moos SP, Stefadouros M, Young DT. Wall thickness and diastolic properties of the Young DT. Wall thickness and diastolic pro

20 Wagner JA, Sax FL, Weisman HF, et al. Calciumantagonist receptors in the atrial tissue of patients with antagonist receptors in the atrial tissue of patients with 320:755-61.

21 Burchell HB. A clinical appraisal of atrial transport function Lancet 1964; i:775-9.

22 Glancy DL, O'Brien KP, Gold HK, Epstein SE. Atria fibrillation in patients with idiopathic hypertrophic subaortic stenosis. Br Heart J 1970;32:652-9.

\section{ABSTRACTS IN CARDIOLOGY}

\section{Variable response to vasodilators in primary pulmonary hypertension}

Rich and colleagues have previously reported the use of high dose calcium-channel blocking therapy for primary pulmonary hypertension and provided evidence for a long-term reduction in pulmonary arterial pressure (Circulation 1987;76:135-41). They now write about the effect of these high doses on survival after five years. It is no surprise that their treated patients did better. They were less ill. Such patients presumably have pulmonary arteries that are more muscular and capable of dilating so that cardiac output can increase and systemic blood pressure is maintained. Rich et al compare their less severely affected patients with the commoner, more severely affected patients who were likely to do badly. The much smaller doses of calcium channel blockers that can be taken by "non-responders" may still be beneficial; but this is harder to prove. Unless they feel considerable benefit patients will not tolerate the painful puffy legs that are an unsightly consequence of high dose treatment with these agents.

Rich et al only gave warfarin to patients with non-uniform perfusion lung scans and they claim that this particularly helped "non-responders". Most patients with primary pulmonary hypertension have uniform lung scans, but soft patchy defects are sometimes seenthough they look quite different from the stark segmental defects seen in patients with major vessel occlusions. I have long believed and advocated the use of long-term warfarin. This may prevent secondary thrombosis in a pulmonary vascular bed with endothelial damage but also has a major role in patients whose very low flows make them vulnerable to venous thromboembolism.

Primary pulmonary hypertension is not uniformly progressive. All patients should have their response to vasodilator drugs evaluated. I believe that calcium-channel blocking agents should be given in the maximum tolerated doses to all patients who will take them, the response to acute administration being a guido both to prognosis and to dose. I also believe that all patients with this diagnosis should be given long-term anticoagulants.

CELIA M OAKI.EY

\section{The effect of high doses of calcium-channel blockers on survival in primary pulmonary hypertension}

\section{Stuart Rich, Elizabeth Kaufmann, Paul S Levy}

\begin{abstract}
Background-Primary pulmonary hypertension is a progressive, fatal disease of unknown cause. Vasodilator drugs have been used as a treatment, but their efficacy is uncertain.

Methods.- We treated 64 patients with primary pulmonary hypertension with high doses of calciumchannel blockers. Patients who responded to treatment (defined as those whose pulmonary-artery pressure and pulmonary vascular resistance immediately fell by more than 20 per cent after challenge) were treated for up to five years. Their survival was compared with that of the patients who did not respond and with patients enrolled in the National Institutes of Health (NIH) Registry on Primary Pulmonary Hypertension. Warfarin was given to $\mathbf{5 5}$ per cent of the patients as concurren therapy, on the basis of a lung scan showing nonuniformity of pulmonary blood flow (47 per cent of patients who responded and 57 per cent of those who
\end{abstract} did not respond)

Results.-Seventeen patients ( 26 per cent) responded to treatment, as indicated by a 39 per cent fall in pulmonary-artery pressure and a 53 per cent fall in the pulmonary-vascular-resistance index $(P<0.001$ ). Nifedipine (mean $[ \pm$ SD] daily dose, $172 \pm 41 \mathrm{mg}$ ) was given to 13 patients, and diltiazem (mean daily dose, $720+208 \mathrm{mg}$ ) was given to 4 patients. After five years, 94 per cent of the patients who responded (16 and 17) were alive, as compared with 55 per cent of the patients who did not respond ( 26 of $47, P=0.003$ ). The survival of the patients who responded was also significantly better than that of the NIH registry cohor $(P=0.002)$ and patients from the NIH registry who were treated at the University of Illinois $(P=0.001)$. The use of warfarin was associated with improved survival $(P=0.025)$, particularly in the patients who did not respond.

Conclusions.-This study suggests that high doses of calcium-channel blockers in patients with primary pulmonary hypertension who respond with reductions in pulmonary-artery pressure and pulmonary vascular resistance may improve survival over a five-year period. (N Engl J Med 1992;327 76-81.) 